'Departamento de Ciencias Neurológicas Oriente, Universidad de Chile. Santiago, Chile.

${ }^{2}$ Servicio de Neurología, Neurocirugía y Neurorradiología, Clínica Santa María. Santiago, Chile.

${ }^{3}$ Servicio de Neurología, Hospita San Borja Arriarán. Santiago, Chile.

${ }^{4}$ Corporación de investigación neurológica de Santiago (CINSAN). Santiago, Chile.

a'Doctor en Ciencias Médicas.

Trabajo no recibió financiamiento. Los autores declaran no tener conflictos de interés.

Recibido el 10 de septiembre de 2019, aceptado el 9 de marzo de 2020.

Correspondencia a: Dr. Pablo Reyes Sánchez Departamento de Ciencias Neurológicas Oriente, Universidad de Chile. Santiago, Chile. preyes@cinsan.org

\section{Hipofisitis linfocitaria primaria. Caso clínico}

\author{
PABLO REYES S. ${ }^{1,2,4, a}$, DANIEL ANDREU ${ }^{2,3}$, CAMILA TOLEDO $^{1}$
}

\section{Primary lymphocytic hypophysitis. Report of one case}

Primary lymphocytic hypophysitis is an autoimmune disease characterized by lymphocytic infiltration of the pituitary gland, with a higher incidence during late pregnancy and the postpartum period. It causes glandular destruction, mass effect and symptoms such headache, visual field defects, ophthalmoplegia and symptoms of hypopituitarism. We report a 38-year-old postpartum woman who, after giving birth presented decreased left visual acuity associated with a non ictal headache. Magnetic resonance imaging demonstrated a sellar mass associated with decreased free thyroxine and cortisol levels. Suspecting a primary lymphocytic hypophysitis, she was treated with prednisone $60 \mathrm{mg} /$ day and hormonal replacement therapy. One month later, size of the pituitary gland decreased, and the visual field defect improved. Steroidal treatment was maintained for 36 months and progressively tapered. After two years of follow-up, the imaging studies show a normal sized pituitary gland.

(Rev Med Chile 2020; 148: 258-262)

Key words: Autoimmune Hypophysitis; Hypopituitarism; Pituitary Gland; Postpartum Period.

\section{$\mathrm{L}$} a hipofisitis linfocitaria primaria (HLP) es una enfermedad autoinmune caracterizada por infiltración linfocitaria difusa de la hipófisis, que puede afectar su porción anterior (adenohipofisitis linfocitaria [AHL]), posterior (infundíbulo neurohipofisitis [INHL]) o ambas (panhipofisitis $[\mathrm{PHL}])^{1}$. Histopatológicamente se subdivide en 6 formas: linfocítica $(71,8 \%)$, granulomatosa $(18,6 \%)$, xantomatosa $(3,3 \%)$, asociada a IgG4 $(1,8 \%)$, necrotizante $(0,7 \%)$ y mixta $(3,7 \%)^{1}$. Su prevalencia fluctúa entre 0,24 y $0,88 / 100.000^{2,3}$, con una incidencia anual probable de 1/9.000.000 de habitantes y representa menos de $0,5 \%$ de las masas selares ${ }^{4,8}$. La AHL es la más frecuente $(65 \%)$ y, característicamente, se presenta en mujeres jóvenes con una razón mujeres:hombres de 6:1 y promedio de edad de $35 \pm 13$ años, durante el último mes del embarazo o dentro de los 2 primeros meses postparto. La INHL (10\%) no tiene predisposición por género; y la PHL
(25\%) es algo más frecuente en mujeres (razón mujeres: hombres de $1,9: 1)^{3}$, siendo estas últimas dos de presentación más tardía $(42 \pm 17 \text { años })^{3}$. La HLP puede provocar síntomas neurológicos como cefalea de inicio gradual o ictal, trastornos del campo visual por compresión del quiasma óptico o diplopía, cuando invade el seno cavernoso ${ }^{1}$.

A continuación, presentamos el caso clínico de una paciente que desarrolló un HLP en el puerperio, destacando la importancia de incluir este cuadro en el diagnóstico diferencial de las masas selares, y también considerarlo en el diagnóstico diferencial de la cefalea en el puerperio.

\section{Caso clínico}

Paciente de sexo femenino de 38 años, sin antecedentes médicos, con ascendencia japonesa, puérpera de 13 días; tras un parto sin complica- 
ciones, evoluciona con cefalea frontal bilateral no ictal, irradiada al ojo izquierdo, alteración visual izquierda e hipogalactia. Evaluada inicialmente por oftalmología con un campo visual de Goldman que mostró una cuadrantopsia heterónima bitemporal superior, mayor en el ojo izquierdo (Figura 1), hospitalizándose para estudio y tratamiento sintomático. En el examen neurológico, solo destacaba alteración del campo visual en el cuadrante superior externo del ojo izquierdo.

Se realizó resonancia magnética (RM) de cerebro y de silla turca, que fue interpretada inicialmente como un macroadenoma hipofisiario que comprimía el quiasma óptico (Figura 2, A, B, C, y D). Dentro del estudio hormonal destacaba TSH
$0.5 \mathrm{uUI} / \mathrm{ml}(0,4-4)$, T4L $0.688 \mathrm{ng} / \mathrm{dL}(0,89-1,76)$, cortisol am $3,9 \mathrm{mg} / \mathrm{dL}$ (5-25), prolactina postparto $10,2 \mathrm{ng} / \mathrm{ml}(1,9-25)$, IGF-1 $226 \mathrm{ng} / \mathrm{ml}(63,4-223)$ y ACTH 5 pg/mL (9-69). No hubo evidencia de diabetes insípida. En el hemograma destacaba leucocitos 7.500/UL y VHS de $46 \mathrm{~mm} / \mathrm{h}$, estudio reumatológico negativo, recuento de subclases de inmunoglobulinas dentro de límites normales y anticuerpos antitiroglobulina y antitiroperoxidasa dentro de rangos normales. Diagnosticado un hipopituitarismo parcial, sumado a las características epidemiológicas e imagenológicas se sospechó una HLP, iniciándose tratamiento con prednisona $60 \mathrm{mg}$ al día y levotiroxina 100 ug/día, con lo que la paciente experimentó una mejoría progresiva

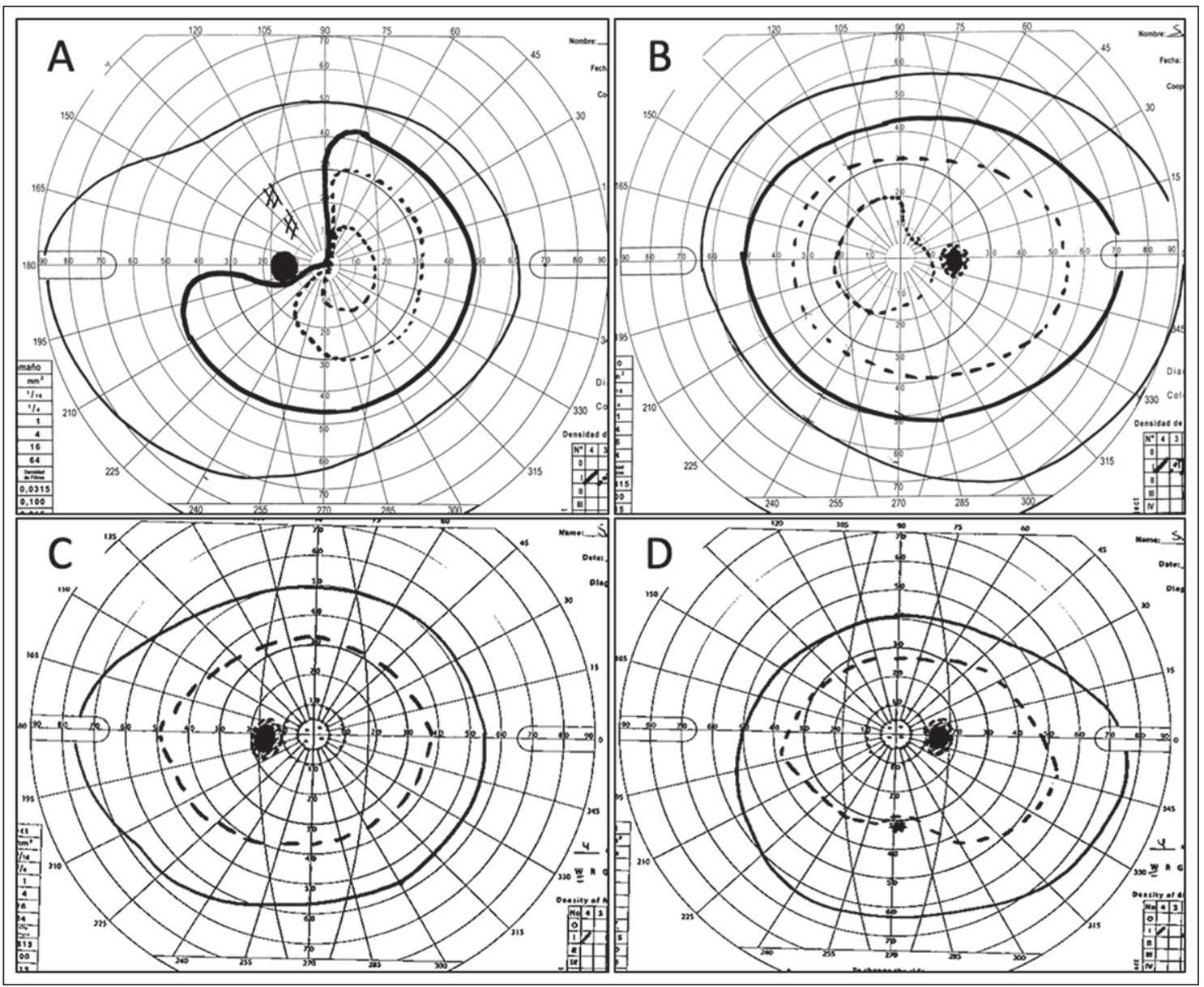

Figura 1. Campo visual de Goldman: A y B, ojo izquierdo y derecho respectivamente al momento del diagnóstico, evidenciando una cuadrantopsia heterónima bitemporal superior, com mayor compromiso en ojo izquierdo. C y D, ojo izquierdo y derecho respectivamente en control 2 meses después del inicio de corticoides. 


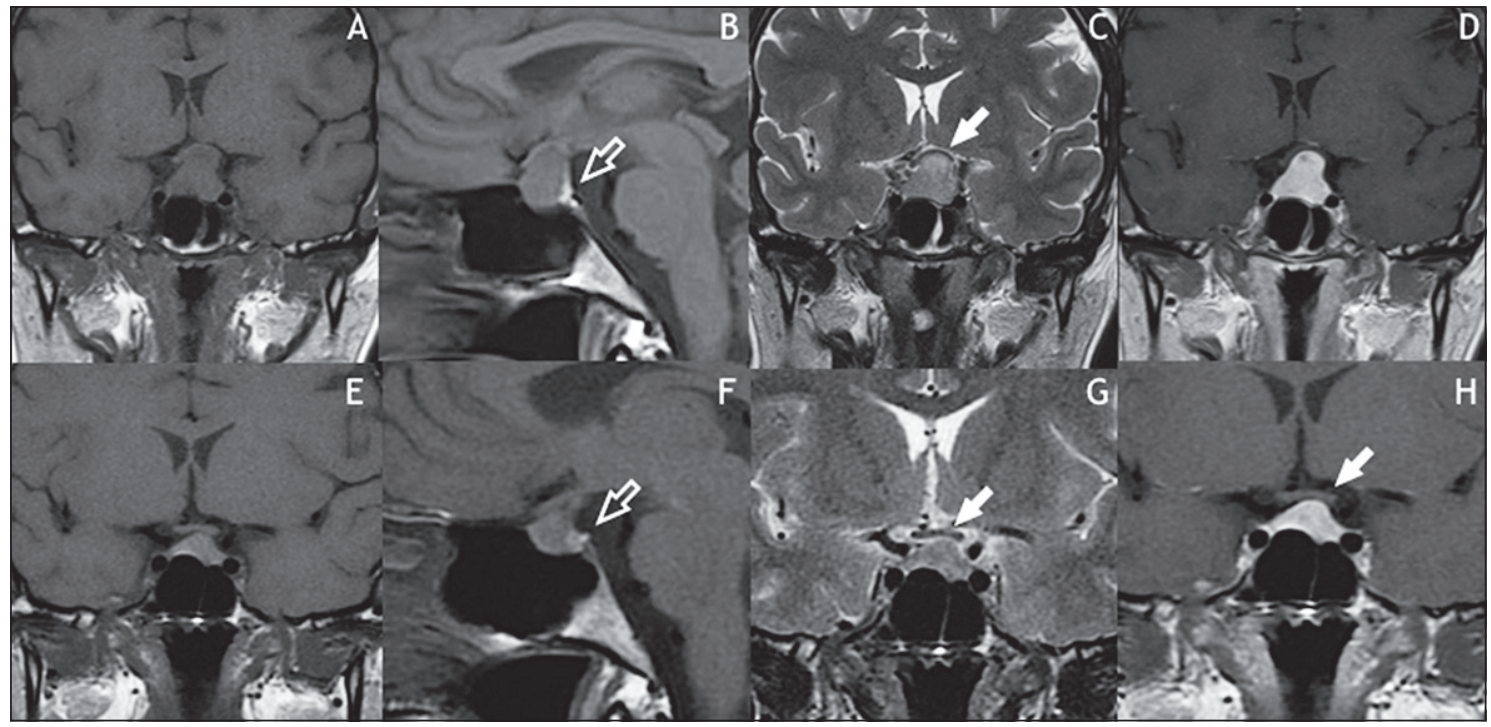

Figura 2. $A, B, C$ y D: Resonancia nuclear magnética (RNM) de silla turca al momento del diagnóstico, muestra un proceso expansivo selar con extensión supraselar que capta intensamente contraste, con neurohipófisis (flecha sin relleno) de características normales. El quiasma óptico (flecha rellena) se encontraba desplazado cranealmente y adelgazado. E, F, G y H son imágenes de la RNM de control un mes después del inicio del tratamiento con corticoides, demostrando una significativa disminución de volumen hipofisiario. A y E: secuencias T1 coronal; B y F: T1 sagital; C y G: T2 coronal y D y H T1 coronal contrastado.

de sus síntomas. Tras un mes hubo reducción del tamaño glandular (Figura 2) y mejoría del campo visual (Figura 1). Tras dos años de evolución, el tamaño y características imagenológicas glandulares se normalizaron. Mantiene tratamiento con levotiroxina. Respecto al tratamiento esteroidal, se mantuvo con prednisona en disminución progresiva por 36 meses hasta suspensión, pero tras 2 meses de suspendido el tratamiento desarrolló una insuficiencia suprarrenal; luego de 1 año y medio de reiniciado el tratamiento, nueva suspensión progresiva que mantiene la fecha.

\section{Discusión}

La baja frecuencia de la HLP y su ambigua presentación clínica e imagenológica plantean dificultades diagnósticas relevantes. Un error diagnóstico puede significar la extirpación de la hipófisis, exponer al paciente a riesgo quirúrgico, a un procedimiento fútil y alteraciones endocrinas permanentes. Desde el punto de vista clínico, las características epidemiológicas como la edad, sexo, embarazo y puerperio, así como la presencia de una masa selar no secretora, con captación de contraste homogéneo, sin remodelación de la silla turca, e hipopituitarismo parcial o total permiten sospechar la $\mathrm{HPL}^{1,3,10}$.

Nuestra paciente tenía los factores de riesgo y las características clínicas típicas de una AHL: mujer, joven, puérpera, ascendencia asiática, síntomas visuales, cefalea con perfil subagudo, hipocortisolismo, hipotiroidismo, ausencia de diabetes insípida, presencia de una masa selar con captación homogénea de contraste y buena respuesta al tratamiento médico ${ }^{1,3,10}$.

El cuadro clínico no es exclusivo de la HLP, compartiendo características con pacientes con adenomas hipofisiarios y síndrome de Sheehan. La ausencia de hemorragia en el parto, hipotensión o shock hacen menos probable un síndrome de Sheehan. El perfil del compromiso hormonal puede ayudar a sospechar la presencia de una HLP en lugar de un adenoma hipofisiario. Alrededor de $78 \%$ de los pacientes con AHL tienen algún déficit hormonal ${ }^{11}$, a diferencia de los pacientes con adenoma hipofisiario, que sufren más frecuentemente hipersecreción glandular (63-73\% $)^{9,12}$. Por otra parte, el perfil de déficit hormonal difiere, en 
cuanto la AHL tiene más frecuentemente déficit de ACTH (61\%), TSH (45\%), LH/FSH (39\%), prolactina $(36 \%)$ y GH $(36 \%)^{13}$, mientras que los pacientes con macroadenomas hipofisiarios no funcionantes tienen con mayor frecuencia déficit de GH (85\%), LH/FSH (61\%), TSH (32\%), ACTH $(31 \%)$ y prolactina $(22 \%)^{14}$. Dado esto, la presencia de compromiso corticotropo y tirotropo, con normalidad del eje somatotropo es un elemento de sospecha de AHL.

Se ha demostrado la existencia de anticuerpos antipituitarios (AAP), sin que tengan un rol patogénico claro ${ }^{16}$. Algunos de los posibles autoantígenos descritos son la hormona de crecimiento, la hormona de crecimiento placentaria ${ }^{7}$, alfa enolasa, CGI99, somatomamotropina coriónica, factores pituitarios específicos la y 2 y secretogranina $2^{1,5,7,16}$. La sensibilidad de los AAP alcanza $68 \%$ para la AHL, $33 \%$ para la INHL y $80 \%$ para la PHL, dependiendo del método utilizado ${ }^{3}$. Sin embargo, estos pueden estar presentes en deficiencias hormonales aisladas (ACTH 40\%, LH/FSH $38 \%$, GH $15 \%$, TSH $16 \%)^{5,6}$, otras enfermedades autoinmunes y tumores hipofisiarios ${ }^{5,17}$, por lo que no se consideran de utilidad clínica.

El principal diagnóstico diferencial radiológico es con el adenoma hipofisiario. En la RM sin contraste los pacientes con AHL presentan una glándula simétrica y homogéneamente ensanchada, engrosamiento de tallo hipofisiario y una silla turca normal; mientras que los macroadenomas hipofisiarios muestran una glándula asimétrica y heterogénea con áreas quísticas, hemorrágicas o ambas, desplazamiento del tallo hipofisiario, alteración del suelo de la silla e infrecuentemente alteración de la señal de la neurohipófisis ${ }^{3}$. Con contraste, la AHL muestra una captación intensa y homogénea, mientras que los adenomas hipofisiarios una captación menos intensa y enlentecida $^{3}$. La presencia de las siguientes características clínico radiológicas: síntomas durante el final del embarazo o en el puerperio, edad $\leq 30$ años, volumen glandular pequeño, una glándula simétrica con intensa captación de contraste, pérdida de la señal neurohipófisis y engrosamiento del tallo hipofisiario, permiten construir una escala con una sensibilidad, especificidad, valor predictivo positivo y valor predictivo negativo de $92 \%, 99 \%$, 97\% y 97\%, respectivamente, para el diagnóstico de HLP vs adenoma no funcionante ${ }^{18}$.

La forma primaria de hipofisitis es idiopática, mientras que las formas secundarias incluyen enfermedades sistémicas de origen autoinmune, infecciosas, tumores selares y paraselares, síndromes paraneoplásicos y fármacos, destacando los inhibidores de checkpoint (CTLA4, PD1 y PD1/ PD1-ligando $)^{1,19}$.

El objetivo del tratamiento agudo es resolver los síntomas compresivos y prevenir una insuficiencia adrenal aguda, el tratamiento crónico es restituir las funciones hormonales ${ }^{1}$. La terapia médica de primera línea son los glucocorticoides, la segunda línea es la azatioprina, metotrexato y ciclosporina-A, dejando como tercera línea a rituximab o infliximab ${ }^{3}$. No existe consenso en cuanto a las dosis ni prolongación del tratamiento, aunque es frecuente el uso de prednisona 30-40 mg día por 2 a 4 semanas y luego descenso en 2 a 6 meses $^{3}$. Se describe una mejoría de $62,5 \%$ con dosis de prednisona $\geq 10 \mathrm{mg} /$ día y de $44,4 \%$ con dosis $\leq 7,5 \mathrm{mg} /$ día. El tratamiento con prednisona $50 \mathrm{mg} /$ día en dosis decrecientes durante 13 meses comparado con ausencia de tratamiento (observación), mejoró la función hipofisiaria en 58,3\% vs $25 \%(p=0,04)^{19}$. El uso de metilprednisolona en dosis decrecientes durante 6 meses redujo el tamaño glandular en $77 \%$ de los pacientes ${ }^{8}$. La recurrencia tras la suspensión de los glucocorticoides se reporta en hasta $38 \%{ }^{14}$.

El tratamiento quirúrgico se reserva para síntomas compresivos significativos, déficit campimétrico, disminución de agudeza visual, y cuando el diagnóstico preoperatorio no ha sido concluyente $e^{3}$. En $68-100 \%$ de los pacientes se logra una disminución del volumen glandular, y en $78 \%$ mejoría del campo visual, con una recurrencia de hasta $20 \%{ }^{14}$. El $45 \%$ de los pacientes mejora su función hormonal ${ }^{3}$. Entre los efectos adversos, se han reportado deterioro de la función hipofisiaria (11-40\%), meningitis y fístula de líquido cefalorraquídeo. La radioterapia y radiocirugía (gamma-knife) se han utilizado en falla al tratamiento tanto médico como quirúrgico, sin que exista consenso en la dosis total ${ }^{3,14}$.

El seguimiento de los pacientes demuestra que $73 \%$ de los pacientes requiere reemplazo hormonal permanente, $16 \%$ mejora luego del tratamiento de reducción de la glándula sin requerir tratamiento hormonal posterior, $3 \%$ resuelve de forma espontánea y $8 \%$ fallece, presumiblemente por falla adrenal aguda ${ }^{3}$.

Nuestra paciente no requirió tratamiento qui- 
rúrgico, ya que el tratamiento médico fue efectivo y se restableció la función glandular. Destaca el desarrollo de insuficiencia suprarrenal luego de la suspensión progresiva de corticoides.

Agradecimientos: Los autores agradecen el apoyo y asesoría de E.U. Andrea Ampuero en el desarrollo y culminación de este caso clínico.

\section{Referencias}

1. Fukuoka H. Hypophysitis. Endocrinol Metab Clin North Am 2015; 44 (1): 143-9.

2. Fehn M, Sommer C, Ludecke DK, Plockinger U, Saeger W. Lymphocytic hypophysitis: light and electron microscopic findings and correlation to clinical appearance. Endocr Pathol 1998; 9: 71-8.

3. Caturegli P, Newschaffer C, Olivi A, Pomper M, Burger P, et al. Autoimmune hypophysitis. Endocr Rev 2005; 26: 599-614.

4. Buxton N, Robertson I. Lymphocytic and granulocytic hypophysitis: a single centre experience. Br J Neurosurg 2001; 15: 242-6.

5. Caturegli P, Lupi I, Landek-Salgado M, Kimura H, Rose NR. Pituitary autoimmunity: 30 years later. Autoimmun Rev 2008; 7: 631-7.

6. Hashimoto KI, Yamakita N, Ikeda T, Matsuhisa T, Kuwayama A, Sano T, et al. Longitudinal study of patients with idiopathic isolated TSH deficiency: possible progression of pituitary dysfunction in lymphocytic adenohypophysitis. Endocr J 2006; 53: 593-601.

7. Takao T, Nanamiya W, Matsumoto R, Asaba K, Okabayashi T, Hashimoto K. Antipituitary antibodies in patients with lymphocytic hypophysitis. Horm Res 2001; 55 (6): 288-92.

8. Kristof RA, Van Roost D, Klingmuller D, Springer W, Schramm J. Lymphocytic hypophysitis: non-invasive diagnosis and treatment by high dose methylprednisolone pulse therapy? J neurol neurosurg psychiatry 1999; 67: 398-402.

9. Famini P, Maya MM, Melmed S. Pituitary Magnetic
Resonance Imaging for Sellar and Parasellar Masses: Ten-Year Experience in 2598 Patients. J Clin Endocrinol Metab 2011; 96 (6): 1633-41.

10. Falorni A, Minarelli V, Bartoloni E, Alunno A, Gerli R. Diagnosis and classification of autoimmune hypophysitis. Autoinmun Rev 2014; 13 (4-5): 412-6.

11. Hashimoto K, Takao T, Makino S. Lymphocytic Adenohypophysitis and Lymphocytic Infundibuloneurohypophysitis. Endocr J 1997; 44 (1): 1-10.

12. Fernandez A, Karavitaki N, Wass JA. Prevalence of pituitary adenomas: a community-based, cross-sectional study in Banbury (Oxfordshire, UK). Clin Endocrinol (Oxf) 2010; 72 (3): 377-82.

13. Wichers-Rother M, Hoven S, Kristof RA, Bliesener N, Stoffel-Wagner B. Non-functioning pituitary adenomas: endocrinological and clinical outcome after transsphenoidal and transcranial surgery. Exp Clin Endocrinol Diabetes 2004; 112 (6): 323-7.

14. Karaca Z, Kelestimur F. The management of hypophysitis. Minerva Endocrinol 2016; 41 (3): 390-9.

15. Chiloiro S, Tartaglione T, Capoluongo ED, Angelini F, Arena V, Giampietro A, et al. Hypophysitis Outcome and Factors Predicting Responsiveness to Glucocorticoid Therapy: A Prospective and Double-Arm Study. J Clin Endocrinol Metab 2018; 103 (10): 3877-89.

16. Joshi MN, Whitelaw BC, Carroll PV. Mechanisms in endocrinology: Hypophysitis: Diagnosis and Treatment. Eur J Endocrinol 2018; 179 (3): R151-63.

17. Ricciuti A, De Remigis A, Landek-Salgado MA, De Vincentiis L, Guaraldi F, Lupi I, et al. Detection of pituitary antibodies by immunofluorescence: approach and results in patients with pituitary diseases) J Clin Endocrinol Metab 2014; 99 (5): 1758-66.

18. Gutenberg A, Larsen J, Lupi, Rohde V, Caturegli P. A Radiologic Score to Distinguish Autoimmune Hypophysitis from Nonsecreting Pituitary Adenoma Preoperatively. AJNR Am J Neuroradiol 2009; 30 (9): 1766-72.

19. Gubbi S, Hannah-Shmouni F, Verbalis JG, Koch CA. Hypophysitis: An update on the novel forms, diagnosis and management of disorders of pituitary inflammation. Best Pract Res Clin Endocrinol Metab 2019; 101371. doi: 10.1016/j.beem.2019.101371. 